九州大学学術情報リポジトリ

Kyushu University Institutional Repository

\title{
On a kind of duality of multiple zeta-star values
}

Kaneko, Masanobu

Faculty of Mathematics, Kyushu University

Ohno, Yasuo

Department of Mathematics, Kinki University

http://hdl. hand le. net/2324/20433

出版情報: International journal of number theory. 6 (8), pp.1927-1932，2010-12. World Scientific バージョン :

権利関係 : 


\title{
On a kind of duality of multiple zeta-star values
}

\author{
Masanobu Kaneko and Yasuo Ohno
}

September 21, 2009

\section{Main result}

In this note, we prove a certain dulality-type result for height 1 multiple zeta-star values and discuss its possible generalization.

For an index set $\left(k_{1}, k_{2}, \ldots, k_{n}\right)$ of positive integers with $k_{1}>1$, the multiple zeta-star value $\zeta^{\star}\left(k_{1}, k_{2}, \ldots, k_{n}\right)$ is defined by

$$
\zeta^{\star}\left(k_{1}, k_{2}, \ldots, k_{n}\right):=\sum_{m_{1} \geq m_{2} \geq \cdots \geq m_{n}>0} \frac{1}{m_{1}^{k_{1}} m_{2}^{k_{2}} \cdots m_{n}^{k_{n}}} .
$$

If we remove the equality signs in the summation, we obtain the usual multiple zeta value:

$$
\zeta\left(k_{1}, k_{2}, \ldots, k_{n}\right):=\sum_{m_{1}>m_{2}>\cdots>m_{n}>0} \frac{1}{m_{1}^{k_{1}} m_{2}^{k_{2}} \cdots m_{n}^{k_{n}}} .
$$

The height of the multiple zeta or zeta-star value is the number of $k_{i}$ in the index set which is greater than 1 . The following theorem can be regarded as a kind of duality for multiple zeta-star values of height 1.

Theorem 1 For any integers $k, n \geq 1$, we have

$$
(-1)^{k} \zeta^{\star}(k+1, \underbrace{1, \ldots, 1}_{n})-(-1)^{n} \zeta^{\star}(n+1, \underbrace{1, \ldots, 1}_{k}) \in \mathbf{Q}[\zeta(2), \zeta(3), \zeta(5), \ldots],
$$

the right-hand side being the algebra over $\mathbf{Q}$ generated by the values of the Riemann zeta function at positive integer arguments $(>1)$.

Remark For multiple zeta values, there is a well-known duality formula [9], and the height 1 case of the formula reads as

$$
\zeta(k+1, \underbrace{1, \ldots, 1}_{n-1})=\zeta(n+1, \underbrace{1, \ldots, 1}_{k-1})
$$

for $k, n \geq 1$. No such simple formula has been known for multiple zeta-star values. It should be noted that the pair of indices

$$
(k+1, \underbrace{1, \ldots, 1}_{n}) \longleftrightarrow(n+1, \underbrace{1, \ldots, 1}_{k})
$$


in Theorem 1 is different from that in the duality formula for multiple zeta values above.

We can also compute the generating function of the quantity

$$
(-1)^{k} \zeta^{\star}(k+1, \underbrace{1, \ldots, 1}_{n})-(-1)^{n} \zeta^{\star}(n+1, \underbrace{1, \ldots, 1}_{k})
$$

in Theorem 1.

Theorem 2 We have

$$
\begin{aligned}
\sum_{k, n \geq 1}\left((-1)^{k} \zeta^{\star}(k+1, \underbrace{1, \ldots, 1}_{n})-(-1)^{n} \zeta^{\star}(n+1, \underbrace{1, \ldots, 1}_{k})\right) x^{k} y^{n} \\
=\psi(x)-\psi(y)+\pi(\cot (\pi x)-\cot (\pi y)) \frac{\Gamma(1-x) \Gamma(1-y)}{\Gamma(1-x-y)} .
\end{aligned}
$$

Here, $\psi(x)=\Gamma^{\prime}(x) / \Gamma(x)$ is the digamma function, the logarithmic derivative of the gamma function.

\section{Proof of Theorems}

We prove the following basic identity, from which follow both Theorem 1 and Theorem 2. ${ }^{1}$

Proposition For $k, n \geq 1$, we have

$$
\begin{aligned}
& (-1)^{k} \zeta^{\star}(k+1, \underbrace{1, \ldots, 1}_{n})-(-1)^{n} \zeta^{\star}(n+1, \underbrace{1, \ldots, 1}_{k}) \\
= & k \zeta(k+2, \underbrace{1, \ldots, 1}_{n-1})-n \zeta(n+2, \underbrace{1, \ldots, 1}_{k-1}) \\
& +(-1)^{k} \sum_{j=0}^{k-2}(-1)^{j} \zeta(k-j) \zeta(n+1, \underbrace{1, \ldots, 1}_{j}) \\
& -(-1)^{n} \sum_{j=0}^{n-2}(-1)^{j} \zeta(n-j) \zeta(k+1, \underbrace{1, \ldots, 1}_{j}),
\end{aligned}
$$

where we understand an empty sum to be 0.

Proof. We use two formulas for the special value of the function $\xi_{k}(s)$ defined for $k \geq 1$ by

$$
\xi_{k}(s):=\frac{1}{\Gamma(s)} \int_{0}^{\infty} \frac{t^{s-1}}{e^{t}-1} L i_{k}\left(1-e^{-t}\right) d t .
$$

${ }^{1}$ Recently, C. Yamazaki ([8]) gave another proof of them. It uses a generating function of certain sums of multiple zeta-star values which was introduced in [1]. 
In [3], we studied this function and obtained among others the formula

$$
\begin{aligned}
\xi_{k}(n+1)= & (-1)^{k-1}[\zeta(n+1, \underbrace{2,1, \ldots, 1}_{k-1})+\zeta(n+1, \underbrace{1,2,1, \ldots, 1}_{k-1})+\cdots \\
& \cdots+\zeta(n+1, \underbrace{1, \ldots, 1,2}_{k-1})+(n+1) \cdot \zeta(n+2, \underbrace{1, \ldots, 1}_{k-1})] \\
& +\sum_{j=0}^{k-2}(-1)^{j} \zeta(k-j) \cdot \zeta(n+1, \underbrace{1, \ldots, 1}_{j}),
\end{aligned}
$$

where $k, n$ are integers $\geq 1$.

On the other hand, we showed in [6] that the value $\xi_{k}(n)$ is nothing but the multiple zeta-star value of hetight 1 , i.e., we have the formula

$$
\xi_{k}(n+1)=\zeta^{\star}(k+1, \underbrace{1, \ldots, 1}_{n}) .
$$

Since the index sets $(k+1, \underbrace{1, \ldots, 1}_{n-1})$ and $(n+1, \underbrace{1, \ldots, 1}_{k-1})$ are dual (in the context of multiple zeta values) with each other, the main theorem in [6] applied to these index sets with $l=1$ gives the identity

$$
\begin{aligned}
& \zeta(k+2, \underbrace{1, \ldots, 1}_{n-1})+\zeta(k+1, \underbrace{2,1, \ldots, 1}_{n-1})+\zeta(k+1, \underbrace{1,2,1, \ldots, 1}_{n-1})+\cdots \\
& \cdots+\zeta(k+1, \underbrace{1, \ldots, 1,2}_{n-1}) \\
= & \zeta(n+2, \underbrace{1, \ldots, 1}_{k-1})+\zeta(n+1, \underbrace{2,1, \ldots, 1}_{k-1})+\zeta(n+1, \underbrace{1,2,1, \ldots, 1}_{k-1})+\cdots \\
& \cdots+\zeta(n+1, \underbrace{1, \ldots, 1,2}_{k-1}) .
\end{aligned}
$$

Combining (2), (3) and (4), we obtain the proposition. [4]

Proof of Theorems 1 and 2. Recall the formula of Aomoto [2] and Drinfeld

$$
\sum_{k, n \geq 1} \zeta(k+1, \underbrace{1, \ldots, 1}_{n-1}) x^{k} y^{n}=1-\frac{\Gamma(1-x) \Gamma(1-y)}{\Gamma(1-x-y)} .
$$

This together with the standard Taylor expansion of the (logarithm of) gamma function

$$
\Gamma(1+x)=\exp \left(-\gamma x+\sum_{n=2}^{\infty}(-1)^{n} \frac{\zeta(n)}{n} x^{n}\right) \quad(|x|<1, \gamma: \text { Euler's constant })
$$

shows that all multiple zeta values of height 1 (= of type $\zeta(m, 1, \ldots, 1)$ ) can be expressed as polynomials over $\mathbf{Q}$ in the Riemann zeta values. Theorem 1 therefore follows from the formula in Proposition. 
As for the generating series, we start with the formula (5). Replace $k$ with $k+1$ in (5) and divide the both-hand sides out by $x y$, and then differentiate with respect to $x$ and multiply $x y$. Then we obtain

$$
\begin{array}{rl}
\sum_{k, n \geq 1} & k \zeta(k+2, \underbrace{1, \ldots, 1}_{n-1}) x^{k} y^{n} \\
& =-\frac{1}{x}+\frac{\Gamma(1-x) \Gamma(1-y)}{\Gamma(1-x-y)}\left(\frac{1}{x}+\psi(1-x)-\psi(1-x-y)\right),
\end{array}
$$

and hence by interchanging $x$ and $y$ and subtracting, we have

$$
\begin{aligned}
& \sum_{k, n \geq 1}\left(k \zeta(k+2, \underbrace{1, \ldots, 1}_{n-1})-n \zeta(n+2, \underbrace{1, \ldots, 1}_{k-1}) x^{k} y^{n}\right. \\
= & -\frac{1}{x}+\frac{1}{y}+\frac{\Gamma(1-x) \Gamma(1-y)}{\Gamma(1-x-y)}\left(\frac{1}{x}+\psi(1-x)-\frac{1}{y}-\psi(1-y)\right) .
\end{aligned}
$$

Next, by the formula

$$
\sum_{i=2}^{\infty}(-1)^{i} \zeta(i) x^{i-1}=\psi(1+x)+\gamma
$$

(take the logarithmic derivative of (6)) and by (5), we have

$$
\begin{aligned}
& \sum_{k, n \geq 1}(-1)^{k} \sum_{j=0}^{k-2}(-1)^{j} \zeta(k-j) \zeta(n+1, \underbrace{1, \ldots, 1}_{j}) x^{k} y^{n} \\
= & \sum_{i \geq 2, j, n \geq 1}(-1)^{i} \zeta(i) \zeta(n+1, \underbrace{1, \ldots, 1}_{j-1}) x^{i+j-1} y^{n} \\
= & \left(\sum_{i \geq 2}(-1)^{i} \zeta(i) x^{i-1}\right)\left(\sum_{j, n \geq 1} \zeta(n+1, \underbrace{1, \ldots, 1}_{j-1}) x^{j} y^{n}\right) \\
= & (\psi(1+x)+\gamma)\left(1-\frac{\Gamma(1-x) \Gamma(1-y)}{\Gamma(1-x-y)}\right),
\end{aligned}
$$

and thus we obtain

$$
\begin{aligned}
& \sum_{k, n \geq 1}\left((-1)^{k} \sum_{j=0}^{k-2}(-1)^{j} \zeta(k-j) \zeta(n+1, \underbrace{1, \ldots, 1}_{j})\right. \\
& \left.-(-1)^{n} \sum_{j=0}^{n-2}(-1)^{j} \zeta(n-j) \zeta(k+1, \underbrace{1, \ldots, 1}_{j})\right) x^{k} y^{n} \\
& =\left(1-\frac{\Gamma(1-x) \Gamma(1-y)}{\Gamma(1-x-y)}\right)(\psi(1+x)-\psi(1+y)) .
\end{aligned}
$$


By Proposition, Theorem 2 follows from (7), (8), and the standard identities

$$
\psi(1+x)=\frac{1}{x}+\psi(x) \quad \text { and } \quad \pi \cot (\pi x)=\frac{1}{x}+\psi(1-x)-\psi(1+x) .
$$

\section{Possible generalization}

In this section, we propose a possible generalization of Theorem 1 for arbitrary heights.

First, we recall a few notations which are used in [1]. The weight and the depth of multiple zeta-star values $\zeta^{\star}\left(k_{1}, k_{2}, \ldots, k_{n}\right)$ are the sum $k_{1}+k_{2}+\cdots+k_{n}$ and the length $n$ of its index, respectively. We denote by $X_{0}(k, n, s)$ the sum of all multiple zeta-star values of weight $k$, depth $n$ and height $s$, for $k \geq n+s$ and $n \geq s \geq 1$.

Based on the numerical experiments up to weight 11, we conjecture the following.

Conjecture For any integers $k, n \geq s \geq 1$, we have

$(-1)^{k} X_{0}(k+n+1, n+1, s)-(-1)^{n} X_{0}(k+n+1, k+1, s) \in \mathbf{Q}[\zeta(2), \zeta(3), \zeta(5), \ldots]$.

Remark Theorem 1 is nothing but the case when $s=1$ of the above conjecture.

Examples When the weight is 8 and the height is 2 or 3 , we can show (using the double shuffle relations of multiple zeta values) the following identities, which are in favor of the conjecture.

$$
\begin{aligned}
& X_{0}(8,3,2)+X_{0}(8,6,2)=\frac{876}{175} \zeta(2)^{4}-\zeta(2) \zeta(3)^{2}-3 \zeta(3) \zeta(5) \\
& X_{0}(8,4,2)+X_{0}(8,5,2)=\frac{1083}{280} \zeta(2)^{4}+\zeta(2) \zeta(3)^{2}+2 \zeta(3) \zeta(5) \\
& X_{0}(8,4,3)+X_{0}(8,5,3)=\frac{1349}{280} \zeta(2)^{4}-\frac{1}{2} \zeta(2) \zeta(3)^{2}-\zeta(3) \zeta(5)
\end{aligned}
$$

\section{References}

[1] T. Aoki, Y. Kombu and Y. Ohno, A generating function for sums of multiple zeta values and its applications, Proc. Amer. Math. Soc. 136, 387-396 (2008).

[2] K. Aomoto, Special values of hyperlogarithms and linear difference schemes, Illinois J. of Math., 34-2, 191-216 (1990).

[3] T. Arakawa and M. Kaneko, Multiple zeta values, poly-Bernoulli numbers, and related zeta functions, Nagoya Math. J. 153, 1-21 (1999).

[4] V. G. Drinfel'd, On quasitriangular quasi-Hopf algebras and a group closely connected with Gal( $(\overline{\mathbf{Q}} / \mathbf{Q})$, Leningrad Math. J. 2, 829-860 (1991). 
[5] M. Kaneko, A note on poly-Bernoulli numbers and multiple zeta values, Diophantine analysis and related fields (DARF 2007/2008), AIP Conf. Proc. 976, 118-124, Amer. Inst. Phys., Melville, NY, (2008).

[6] Y. Ohno, A generalization of the duality and sum formulas on the multiple zeta values, J. Number Th. 74, 39-43 (1999).

[7] Y. Ohno and D. Zagier, Multiple zeta values of fixed weight, depth, and height, Indag. Math., 12 (4), 483-487 (2001).

[8] C. Yamazaki, On the duality for multiple zeta-star values of height 1, preprint (2009).

[9] D. Zagier, Values of zeta functions and their applications, in ECM volume, Progress in Math., 120 497-512 (1994).

Masanobu Kaneko

Faculty of Mathematics,

Kyushu University,

Motooka, Nishi-ku, Fukuoka 819-0395, Japan.

E-mail: mkaneko@math.kyushu-u.ac.jp

Yasuo Ohno

Department of Mathematics,

Kinki University

Higashi-Osaka, Osaka 577-8502, Japan.

E-mail: ohno@math.kindai.ac.jp 